SCIENTIFIC REPORT

\title{
The pharmacokinetics of linezolid in the non-inflamed human eye
}

\author{
J I Prydal, D R Jenkins, A Lovering, A Watts
}

Br J Ophthalmol 2005;89:1418-1419. doi: 10.1136/bjo.2005.073700

Aim: This study describes the ocular pharmacokinetics of linezolid, an antibiotic with broad spectrum activity against those Gram positive bacteria that are the most frequent cause of postoperative endophthalmitis.

Method: Patients undergoing routine cataract surgery were given a single oral $600 \mathrm{mg}$ dose of linezolid at a variable time before surgery. Aqueous and serum levels of linezolid were assayed by high performance liquid chromatography, and a pharmacokinetic curve constructed from the pooled results.

Results: Orally administered linezolid rapidly achieves levels in the aqueous of non-inflamed eyes that exceed the concentration required to kill Gram positive bacteria (maximum mean concentration 6.8 (SD 1.2) $\mu \mathrm{g} / \mathrm{ml}$ at $2-4$ hours post-dose). An effective concentration is maintained for at least 12 hours, the standard interdose interval for this antimicrobial.

Conclusion: Linezolid offers the possibility of a rapid, oral approach to effective treatment of most cases of postoperative endophthalmitis, with the potential of improving visual outcome.

$\mathrm{P}$ ostoperative endophthalmitis (POE) is an infrequent but sight threatening complication of intraocular surgery. ${ }^{1-3}$ It is most frequently the result of infection with Gram positive bacteria, in particular coagulase negative staphylococci. $^{24}$ The main antibiotic therapy of POE is intraocular inoculation of antibiotics, usually vancomycin and either amikacin or ceftazidime. ${ }^{56}$ Direct intraocular injection is necessary because antibiotic penetration into the eye from the blood stream is restricted by the blood-ocular barrier. The few antibiotics that may achieve therapeutic concentrations in the aqueous and vitreous following systemic administration have mostly Gram negative activity, and cannot reliably treat the Gram positive organisms most frequently implicated in POE. The visual outcome of POE is often poor, despite early aggressive treatment. ${ }^{6}$ An antibiotic with broad spectrum Gram positive activity, which reaches therapeutic concentrations in the eye following intravenous or oral administration, may offer an advance in the early treatment of endophthalmitis, and could result in improved visual outcome.

Linezolid (Pfizer, UK) is the first licensed representative of a new class of antibiotics, the oxazolidinones. ${ }^{7}$ Its spectrum of activity is primarily against Gram positive bacteria, including both methicillin sensitive and resistant staphylococci, enterococci, and Streptococcus pneumoniae. Minimal inhibitory concentration (MIC) values of linezolid against these organisms lie in the range of $1-4 \mu \mathrm{g} / \mathrm{ml}^{8}{ }^{8}$ Linezolid can be administered either orally, with $100 \%$ bioavailability, or intravenously and produces peak serum levels of approximately $18 \mu \mathrm{g} / \mathrm{ml}$ after repeated oral doses of $625 \mathrm{mg}$. In animal studies linezolid reaches concentrations in the eye which are $40 \%$ of serum levels. In human volunteers maximum concentrations of linezolid in cerebrospinal fluid are $70 \%$ of serum levels. ${ }^{7}$ These levels are achieved across a non-inflamed blood-brain barrier, a structure that shares features with the blood-ocular barrier. These findings suggest that linezolid may reach clinically relevant levels in the aqueous and vitreous humours when given either orally or intravenously, and could be a significant advance in the antibiotic therapy of Gram positive endophthalmitis.

Pharmacokinetic studies of ocular antibiotic levels in human cases of endophthalmitis are difficult to perform because of the irregularity and infrequency of presentation of endophthalmitis. Animal models have been used as surrogate representations but give limited information. An alternative approach is to measure antibiotic levels in patients undergoing elective ophthalmic procedures, albeit that generally these patients have non-inflamed eyes and consequently an intact blood-ocular barrier. These patients are likely to represent a worst case scenario in terms of antibiotic penetration into the eye, and are a rigorous test of eye antibiotic levels. This study examines the penetration of linezolid into aqueous humour of patients with non-inflamed eyes undergoing elective cataract surgery and compares it with simultaneous serum levels.

\section{METHOD}

Patients undergoing elective cataract surgery were enrolled in the study. They were given a single oral dose of $600 \mathrm{mg}$ linezolid at a time between 30 minutes and 14 hours before the operation. Otherwise, preparation for surgery proceeded as normal. At the start of surgery a paracentesis was made and $100 \mu \mathrm{l}$ of aqueous humour collected. At the same time $5 \mathrm{ml}$ of venous blood was taken. The interval between dosing and sample collection was calculated. Serum was separated from the blood sample, and both the aqueous fluid and the serum stored at $-70^{\circ} \mathrm{C}$. Assays for linezolid were performed by the antibiotic assay laboratory, Southmead Hospital, Bristol, by high performance liquid chromatography (HPLC) ${ }^{9}$ without knowledge of the dose sample interval. Preliminary tests demonstrated that the risk of false positives by carryover between specimens was negligible. Pharmacokinetic curves were constructed by combining the assay results of individual patients.

This study was approved by University Hospitals of Leicester medical research ethics committee. Patients gave written consent following verbal and written explanation of the purpose, nature, and possible side effects of this study. Patients with known hypersensitivities to linezolid or any excipients of the study drug were excluded. Other exclusion criteria were known abnormal hepatic or renal function, treatment with any drug that inhibits monoamine oxidases A

Abbreviations: HPLC, high performance liquid chromatography; MIC, minimal inhibitory concentration; $\mathrm{POE}$, postoperative endophthalmitis 


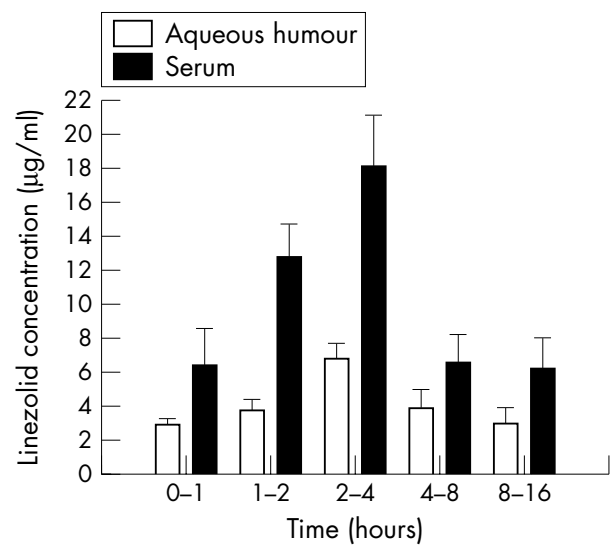

Figure 1 Concentration of linezolid (mean (SD)) in the aqueous humour and serum following a single $600 \mathrm{mg}$ oral dose.

or B within the previous 2 weeks, or serotonin reuptake inhibitors, tricyclic antidepressants, directly and indirectly acting sympathomimetic agents, vasopressive agents, dopaminergic agents, pethidine, or buspirone. Patients with uncontrolled hypertension, phaeochromocytoma, carcinoid, thyrotoxicosis, bipolar depression, schizoaffective disorder, acute confusional states, or a history of linezolid use in the week before surgery were also excluded.

\section{RESULTS}

Thirty four patients, aged $50-93$ years (50\% female) were recruited. The results are given in figure 1 . No adverse effects were recorded. Numbers of patients in the time periods were 0-1 hours, seven; 1-2 hours, 12; 2-4 hours, four; $4-8$ hours, two, and 8-16 hours, seven.

The penetration of linezolid into aqueous humour was rapid, reaching a mean of $3.4(1.9) \mu \mathrm{g} / \mathrm{ml}$ within 1 hour after administration and a maximum concentration $\left(\mathrm{C}_{\max }\right)$ of 6.8 (1.2) $\mu \mathrm{g} / \mathrm{ml}$ at $2-4$ hours. Thereafter, concentrations fell to $3.9(1.6) \mu \mathrm{g} / \mathrm{ml}$ at $4-8$ hours and 2.9 (2.1) $\mu \mathrm{g} / \mathrm{ml}$ by $8-$ 16 hours.

\section{DISCUSSION}

We have characterised the penetration profile of linezolid into the aqueous humour of non-inflamed human eyes after a single oral dose and compared this with simultaneous measurements of plasma concentration. The results indicate that concentrations of linezolid sufficient to have antimicrobial activity against the vast majority of organisms causing POE are achieved within 60 minutes of oral administration of a standard single dose of $600 \mathrm{mg}$. The linezolid concentrations remain above the MIC for most Gram positive bacteria for at least 12 hours, the usual interdose interval. The only major exception are those enterococci with an MIC of $4 \mu \mathrm{g} / \mathrm{ml}$, but these are responsible for only a small proportion of infections. ${ }^{4}$ Concentrations of $4 \mu \mathrm{g} / \mathrm{ml}$ were exceeded in the 2-4 hour interval.

Linezolid exhibits time dependent antimicrobial activity ${ }^{10}$ - that is, clinical effectiveness is determined by the proportion of time between doses that the concentration of linezolid at the site of infection exceeds the MIC. Our study indicates that for most Gram positive infections, this proportion approaches $100 \%$ in the aqueous humour after a single dose.

Our findings are supported by those of Garcia Vazquez et al, ${ }^{11}$ who reported aqueous concentrations of linezolid of $4.9 \mu \mathrm{g} / \mathrm{ml}$ at 1 hour following a $600 \mathrm{mg}$ intravenous dose, rising to $5.2 \mu \mathrm{g} / \mathrm{ml}$ at $135-270$ minutes post-dose. Fiscella et $a l^{12}$ found that aqueous concentrations exceeded $6 \mu \mathrm{g} / \mathrm{ml}$ in the first 2 hours after a second $600 \mathrm{mg}$ oral dose. However, neither of these studies had a sample regimen that extended over the full dose interval. In our study we have been able to show that the good concentrations seen by Garcia Vazquez and Fiscella in the first 4 hours after dosing are continued throughout the whole dose interval.

Our results were from non-inflamed eyes. The pharmacokinetics of drug movement across the blood-ocular barrier is complicated. Inflammation may increase penetration and, if this were the case for linezolid, our results would represent the lower end of the concentration range to be found in infected eyes.

We have shown good penetration of linezolid into the aqueous, but as POE also involves vitreal infection further work is necessary to establish the clinical value of the drug in this situation. Set against the potential benefit of an effective antibiotic in a sight threatening condition is the risk of the emergence of linezolid resistance. However, endophthalmitis is rare and unlikely to be a major contributor to drug resistance on a global scale.

\section{Authors' affiliations}

J I Prydal, Department of Ophthalmic Surgery, University Hospitals of Leicester NHS Trust, Leicester LE1 5WW, UK

D R Jenkins, Department of Clinical Microbiology, University Hospitals of Leicester NHS Trust, Leicester LE1 5WW, UK

A Lovering, Bristol Centre for Antimicrobial Research and Evaluation, Southmead Hospital, Bristol BS10 5NB, UK

A Watts, Department of Ophthalmic Surgery, University Hospitals of Leicester NHS Trust, Leicester LE1 5WW, UK

Competing interests: Pfizer UK paid for the drug assays in this study and provided research funds to University Hospitals of Leicester NHS Trust. JIP and AW have been reimbursed by Pfizer for attending one conference.

Correspondence to: Jeremy Prydal, Department of Ophthalmic Surgery, University Hospitals of Leicester NHS Trust, Infirmary Square, Leicester LE1 5WW, UK; iip@prydal.co.uk

Accepted for publication 15 July 2005

\section{REFERENCES}

1 Javitt JC, Vitale S, Canner JK, et al. National outcomes of cataract extraction. Endophthalmitis following in-patient surgery. Arch Ophthalmol $1991 ; 109: 1085-9$.

2 Fisch A, Salvanet A, Prazuck T, et al. Epidemiology of infective endophthalmitis in France. Lancet 1991;338:1373-6.

3 Norregaard JC, Thoning H, Bernth-Petersen P, et al. Risk of endophthalmitis after cataract extraction: results from the International Cataract Surgery Outcome study. Br J Ophthalmol 1997;81:102-6.

4 Han DP, Wisniewski SR, Wilson LA, et al. Spectrum and susceptibilities of microbiologic isolates in the endophthalmitis vitrectomy study. Am J Ophthalmol 1996;122:1-17.

5 Endophthalmitis Vitrectomy Study Group. Results of the endophthalmitis vitrectomy study. A randomized trial of immediate vitrectomy and of intravenous antibiotics for the treatment of postoperative bacterial endophthalmitis. Arch Ophthalmol 1995;113:1479-96.

6 Okhravi N, Towler HMA, Hykin P, et al. Assessment of a standard treatment protocol on visual outcome following presumed bacterial endophthalmitis. Br J Ophthalmol 1997;81:719-25.

7 Clemett D, Markham A. Linezolid. Drugs 2000;59:815-28.

8 Johnson AP, Warner M, Livermore DM. Activity of linezolid against multiresistant Gram-positive bacteria from diverse hospitals in the United Kingdom. $J$ Antimicrob Chemother 2000;45:225-30.

9 Lovering AM, Zhang G, Bannister GC, et al. Penetration of linezolid into bone, fat, muscle and haematoma of patients undergoing routine hip replacement. J Antimicrob Chemother 2002;50:73-77.

10 MacGowan AP. Pharmacokinetic and pharmacodynamic profile of linezolid in healthy volunteers and patients with Gram-positive infections. J Antimicrob Chemother 2003;51(Suppl S2):ii17-25.

11 García Vázquez E, Mensa J, López Y, et al. Penetration of linezolid into the anterior chamber (aqueous humour) of the human eye after intravenous administration. Antimicrob Agents Chemother 2004;48:670-2.

12 Fiscella RG, Lai WW, Buerk B, et al. Aqueous and vitreous penetration of linezolid (Zyvox) after oral administration. Ophthalmology 2004;111:1191-5. 